\title{
Correction to: A Multi Material Shell Model for the Mechanical Analysis of Triaxial Braided Composites
}

\author{
A. García-Carpintero ${ }^{1,2} \cdot$ M. Herráez ${ }^{1,2} \cdot$ J. Xu $^{1} \cdot$ C. S. Lopes ${ }^{1} \cdot$ C. González ${ }^{1,2}$ \\ Published online: 20 November 2018 \\ (C) Springer Nature B.V. 2018
}

\section{Correction to: Appl Compos Mater (2017) 24:1425-1445 \\ https://doi.org/10.1007/s10443-017-9593-9}

The original version of this article unfortunately requires correction with respect to the affiliation of some of the co-authors. The corrections are as follows:

1. The co-authors "A. García-Carpintero • M. Herráez" should be affiliated to both affiliations 1 (IMDEA Materials Institute) and 2 (Universidad Politécnica de Madrid). Their correct affiliations are now shown here.

2. The institution's name of affiliation 2 "Polytechnic University of Madrid" should be "Universidad Politécnica de Madrid".

The online version of the original article can be found at https://doi.org/10.1007/s10443-017-9593-9

C. González

c.gonzalez@upm.es

1 IMDEA Materials Institute, Tecnogetafe, c/Eric Kandel, 2, 28906 Madrid, Spain

2 Department of Materials Science, Universidad Politécnica de Madrid, ETS de Ingenieros de Caminos, 28040 Madrid, Spain 